\title{
CARRETERAS Y CAMINOS ALICANTINOS EN EL PRIMER TERCIO DEL S IGLO XX
}

\author{
Jesús Rafael de Vera Ferre
}

\section{RESUMEN}

Una vez finalizados en la centuria anterior los ejes básicos de la malla de carreteras de la provincia de Alicante, es pecialmente los que unen a la ciudad capital con Alcoy, La Marina, la meseta y Orihuela, la política que se va a seguir en este campo durante el primer tercio del siglo XX en el Sur del País Valenciano va a venir marcada, como fiel reflejo de lo que ocurre a escala estatal, por dos líneas básicas de actuación: mejorar cualitativamente las rutas más importantes y adensar la red de caminos vecinales, creando un complejo sistema capilar de vías que conectan el ferrocarril y los puertos con zonas geográficas cada vez más alejadas, lo que facilita las transformaciones de los paisajes agrarios y un cambio positivo en el proceso industrializador, a la vez que ayuda a la cristalización del capitalismo y a la creación de un auténtico mercado interior, mientras que la red de ciudades se consolida, hecho que se hace especialmente patente en las principales encrucijadas.

\section{ABSTRACT}

Once the main targets in the province of Alicante road networks had been achieved during the pevians century, especially those linking the city capital with Alcoy, la Marina, la Meseta and Orihuela, the policy followed in this field in the first third of century in the South of the Valencian Region are determined, as a miror like reflection of what is happening in the rest of the country, by two fundamental lines of action: to improve the quality of the most important routes and increase the side road system, building a complex web of roads linking the railway and ports with the more distant geographical areas. This produces changes in the agricultural backgrounds and a positive improvement in the industrializing process, and helps to root capitalism crealing a real home market, cohile the city and town network consolidates being this fact especially obvions is the main crossroads. 


\section{El significado de la política de carreteras durante el período alfonsino}

La provincia de Alicante vive profundas transformaciones económicas y sociales durante el reinado de Alfonso XIII, fiel reflejo de las acaecidas en el sector agrario y de la creciente inversión de los beneficios procedentes del mismo y del comercio en la industria ${ }^{1}$.

La génesis de estas convulsiones hay que buscarla en parte en las mejoras introducidas en los sistemas de transporte terrestre que se hacen especialmente patentes desde el período isabelino y que, teniendo como motor de desarrollo básico al ferrocarril, se extienden también a la red de carreteras y caminos, coadyuvando de este modo a introducir el territorio dentro de los circuitos capitalistas de la mano de la clase burguesa.

A la postre, la preocupación por las obras públicas durante la época alfonsina, que alcanza su cumbre durante la dictadura primorriverista, hunde sus raíces en el intento de conseguir consolidar, ya de forma definitiva, un mercado interior, tal como se venía alentando por influencia de los prohombres liberales en los aparatos del poder desde la centuria anterior. Es significativo al respecto el hecho de que el Plan de Caminos Provinciales de 1926 ponga un especial énfasis en primar la construcción de aquellas vías que enlacen centros de producción, transporte o consumo, tales como estaciones ferroviarias, puertos, embarcaderos y mercados.

El impulso dado a estas rutas hay que enmarcarlo además en un doble contexto: el mantener una política de prestigio en un régimen autoritario, para que sea tildado de eficaz por las clases sociales que lo están manteniendo, y el intentar, por otro lado, aumentar la rentabilidad de las líneas férreas que, por carencias importantes del mercado, presentaban, a veces, serios problemas de capitalización.

Se respondía así a las imperiosas necesidades de generar beneficios y, por ende, de acumular capital, para su posterior inversión en nuevos negocios, de un conglomerado de clases dominantes, basadas en la aristocracia terrateniente y en una burguesía, ora agromercantil, ora industrial, que poseían los resortes del poder provincial, sintiénd ose respaldadas frente a la creciente tensión social por la Dictadura que amp araba de forma eficaz sus intereses.

Paulatinamente, los beneficios obtenidos y los cambios tecnológicos y de demanda de mercado habidos desembocarán en la consolidación de unas cap as burguesas industriales que ven florecer sus negocios al socaire de las mejoras de comunicación y del crecimiento de las industrias agrarias y de bienes de consumo.

Las cambiantes condiciones económicas se verán reflejadas en crisis periódicas y en un enrarecimiento del mercado de trabajo, con bolsas crecientes de paro que se intentarán paliar transfiriendo el problema a la superestructura estatal y corporativa provincial con un relanzamiento en el ramo de las obras públicas, especialmente ostensible en el campo de la construcción de las rutas

${ }^{1}$ Vid. NADA L, J. «Industrialización y desindustrialización del Sureste Español 1817-1913», Moneda y Crédito, 120, 1972, pp. 3-80. 
terrestres. Este intento no es suficientemente extenso como para resolver definitivamente el enervamiento social, por lo que la emigración al Norte de Á frica sirve de aliviadero a la creciente tensión política que amenaza con incardinarse en un proceso revolucionario irreversible que ponga fin a los beneficios obtenidos por las clases dirigentes en los últimos setenta años.

Al finalizar la Dictadura es notoria la estrecha relación existente entre la ralentización de las obras públicas y el aumento de las cotas de paro y radicalización de la vida provincial y española.

Por otro lado, en las obras realizadas se intenta generar el menor gasto posible para poder así multiplicar el número de las acometidas y aportar de esta forma al torrente mercantil capitalista nuevas zonas de producción provinientes de áreas agrarias deficientemente comunicadas hasta el momento.

Al tiemp o que se invierte en las zonas rurales se busca mejorar cualitativamente los grandes circuitos arteriales de la red, construidos en el Ochocientos, para hacer más fluidos los contactos entre la zona litoral y portuaria y el postpaís interior.

Se teje así el apogeo de un proceso que ya se había insinuado levemente en el fugaz Trienio Liberal, alcanzando gran dinamismo durante la época isabelina y la Restauración, cual es el de crear y consolidar una malla de carreteras que respondiera a las necesidades de aumento de los beneficios de las clases preeminentes con el fin de rentabilizar las cuantiosas inversiones llevadas a cabo en el ferrocarril y promover, a la par, la renovación de la agricultura, con la introducción de monocultivos de alta ganancia que desembocaría, a medio plazo, en un proceso industrializador de algunas áreas provinciales.

El techo a la renovación de la red de carreteras y caminos provinciales vino marcado por los graves problemas presupuestarios que venían atravesando las economías locales, que se tradujo, en la praxis, en la creación de una malla que distaba mucho de ser óptima, presentando tramos inconclusos, lo que le restaba coherencia, y déficits en su extensión y densidad, amén de fuertes deficiencias cualitativas en lo concerniente al trazado, obras de fábrica, anchura y conservación.

\section{La red viaria al servicio de las transformaciones económicas}

\subsection{Los cambios en los paisajes agrarios}

El proceso de transformación capitalista de la economía provincial, al que sirvió la creación y mejora de la red viaria de la zona, al socaire de la malla ferroviaria, supuso una creciente tecnificación y modernización de la agricultura alicantina que veía ensanchar sus posibilidades de acceso a la comercialización. Así, por ejemplo, en la Vega Baja del Segura, se estaban viviendo grandes mutaciones en este camp o, alentadas en buena medida por la facilidad natural de la comarca para las comunicaciones, debido a su morfología y a ser zona fronteriza con Murcia, a la vez que se facilitaba el cambio p or la existencia de una imp ortante concentración de la propiedad. Todo ello determina una afluencia 
notable de capitales para la reconversión de los cultivos, que se traduce, entre otros casos, en la transformación de veinticinco mil hectáreas de secano en regadío en la Murada en 1925².

Similar situación se produce en el Marquesado, donde, al igual que ocurriera con la expansión del mercado de la pasa en la centuria anterior, la modernización de las comunicaciones supone en las primeras décadas del Novecientos un aliciente para emprender las costosas obras de regadío tendentes a aumentar la sup erficie cultivada de agrios que, al estar destinados en buena parte de su producción a la exp ortación, necesitaban imperiosamente de un mejoramiento de las conexiones con la red portuaria y con la colindante comarca de la Safor. En los años veinte hay una gran expansión del naranjo en las zonas más bajas del territorio que exp lica, no solamente las cuantiosas inversiones en carreteras en la misma, sino también que se hubiera inaugurado en 1915 la línea férrea, de vía estrecha, que une Denia con la capital lucentina.

La burguesía agraria de la Marina Alta fomenta y reclama constantemente inversiones en transp ortes que hacen mejorar notablemente sus beneficios económicos. El camp esinado vive momentos de convulsión producidos por el abandono parcial de los cultivos tradicionales, mientras que fuertes sumas de dinero se invierten en la extensión del regadío por los llanos aluviales donde cultivos muy seleccionados y de fácil comercialización aseguran fuertes dividendos a sus propietarios y promotores a la par que se agiliza el negocio de los intermediarios.

Por el contrario, a principios del Novecientos la sup erficie dedicada al cultivo de la vid disminuye de manera radical a nivel de toda la provincia, hecho que hunde sus raíces en la llegada de la filoxera, la producción desmesurada de vino y las dificultades de comercialización de los caldos en otros mercados alternativos a los galos. De esta forma, uno de los móviles que habían alentado el crecimiento de la red viaria en la Restauración pierde peso esp ecífico a la hora de programar mejoras en las rutas del Sur del País Valenciano.

\subsection{Los cambios industriales}

Paralelamente a las transformaciones agrarias, se produce un lanzamiento de la industria en la provincia. La distribución espacial de la misma quedaba reflejada por Rafael Altamira:

«No hay en la provincia más centro fabril á la moderna que el de Alcoy (paños y papel de fumar, especialmente). En la capital comienzan ahora á desarrollarse altas industrias como la de fundición de hierro, pero sin que todavía alcancen gran importancia. En cambio, hay en muchos pueblos industrias domésticas de gran valor económico (alpargatería, zapatería, trabajos de esparto, esterería) y otras agrícolas (vinos, aguardientes, pasas) que producen gran riqueza.» ${ }^{3}$ t. 11, p. 311 .

2 RAMOS, V. Historia de la provincia de Alicante y de su capital. Alicante, Diputación Provincial, 1971,

3 ALTAMIRA, R. Derecho consuetudinario y econo mía popular de la provincia de Alicante. Madrid, Imprent a del Asilo de Huérfanos, ed. facs. del Instituto de Estudios Juan Gil Albert, Diputación Provincial, 1985, p. 47. 
Tal como nos indica el texto, fechado en 1903, la estructura industrial de la provincia descansaba en empresas pequeñas y medianas que poseían una cierta dispersión geográfica en su localización, lo que no obsta para que un número elevado de ciudades, todas ellas bien enclavadas en la malla circulatoria, tuvieran una may or configuración industrial. Tal es el caso de Denia en el Marquesado; de Alcoy y Cocentaina en las áreas interiores montañosas; de Villena, Elda, Petrer, Monóvar, Novelda, Elche y Crevillente en el Vinalopó; de Ibi en la Hoya de Castalla; y de Jijona y la capital en el Campo de Alicante. Entre las actividades productivas y extractivas destacan el textil, papelera, juguetera, metalúrgica, marmolera, explotación de salinas, conservas vegetales, alpargatera y calzado ${ }^{4}$.

Sin embargo, la preocupación de las fuerzas políticas p or servir a los intereses de la creciente industria mediante un fortalecimiento de las comunicaciones no va a ser predominante durante todo el primer tercio del siglo XX. Solamente, con el advenimiento de la II República se modificará parcialmente el objetivo básico de la Corporación Provincial en este campo, que seguía siendo fundamentalmente el servicio a los intereses del agro, del comercio y del máximo rendimiento de la estructura ferroviaria, una vez que en la centuria anterior se habían finalizado los principales ejes viarios.

\section{Crecimiento demográfico, expansión urbana y malla circulatoria}

Dada la trayectoria de la economía provincial y la estructura de las redes ferroviarias y de carreteras insertas en la misma, el desarrollo industrial era previsible en la zona, especialmente en lo referente a los bienes de consumo. A ello contribuía también la creciente complejidad del entramado urbano y el crecimiento de la población lo que supone, no solamente una mayor demanda de los productos manufacturados, sino también una transformación cuantitativa y cualitativa de las afluencias circulatorias.

La provincia pasa de 470.149 habitantes a principios de la centuria a 545.838 treinta años después, mientras que la capital crece en más de veinte mil moradores, pasando de 50.142 a 73.071 en igual período 5 .

El mayor incremento demográfico de la ciudad lucentina denota el hecho de que ésta se ha consolidado ya como el principal centro del transporte, del ap arato burocrático y del comercio, debido, en gran medida, a que la red ferroviaria y los itinerarios básicos de las carreteras convergen hacia la misma y sus instalaciones portuarias, actuando como estructurador

4 En 1916, en Elda, existe ya un importante predominio de la industria zapatera, según Guía Comercial de Alicante, Novelda, Monóvar, Elda y Villena. Alicante, 1916. Por su parte, en Elche se hacían entre 4.000 y 5.000 pares de alpargatas anuales, según IBARRA y RUIZ, P. Historia de Elche. Alicante, Tip. V. Botella, 1895, pp. 293-300.

${ }^{5}$ GOZÁLVEZ, V. «La población», apud LÓPEZ GÓMEZ, A. et al. Geografía de la provincia de Alicante. Alicante, Diputación Provincial, 1978, p. 189. 
y centralizador del territorio y de sus flujos circulatorios, lo que genera una reactivación de los negocios mercantiles y de las esp eculaciones inmobiliarias, a las que va a servir, en buena parte, la red tranviaria comarcal.

Aunque la ciudad capital acumula los may ores beneficios dimanantes de las mejoras en el transporte, otros núcleos urbanos se ven también beneficiados de la may or movilidad general en el territorio, especialmente aquellos situados en las principales encrucijadas, que ven reforzada de esta forma su influencia sobre su alfoz, renovando su papel de mercados comarcales.

Las ciudades más favorecidas son las que dominan, dada su situación, varios ramales del trazado arborescente de la malla circulatoria. Tal es el caso, en el Alto Vinalopó, de Villena, mientras que en la zona media del citado corredor florecen Elda y Petrer y, en menor medida, Monóvar, Sax, Novelda y Aspe. En el Bajo Vinalopó, lo hacen Elche y Crevillente y, en la colindante Vega Baja del Segura, Orihuela, Callosa y Torrevieja. En el interior montuoso, despegan Alcoy y Cocentaina, mientras que en las Marinas, lo hacen Denia, Pego y Villajoy osa.

Dada la situación de las ciudades citadas en los ejes circulatorios, ferroviarios y de carreteras, de gran movilidad en el momento, no hay duda de que la complejidad de la red, por sucesivos crecimientos y adensamientos de la misma, va a generar un aumento del poder de atracción de personas, capitales, actividades y tráfico en dichos núcleos.

\section{Directrices básicas de la política viaria a nivel nacional}

En el reinado alfonsino la preocupación por las carreteras es un hecho a nivel nacional, como fiel reflejo de lo que ocurre en el resto del continente, donde desde el Congreso de Carreteras celebrado en París en 1908, se asiste a un creciente interés por ampliar, normalizar y homogeneizar los criterios sobre el tema.

El interés p or los caminos rurales data de los primeros años del reinado de Alfonso XIII ${ }^{6}$. Las Reales Ordenes de 13 de agosto y de 5 de septiembre de 1903 disponen la formación de un Plan de Caminos Vecinales para todo el Estado. La Diputación alicantina se obligó a sufragar el 52 por ciento del coste del mismo en su territorio ${ }^{7}$. Por su parte, el Estatuto Provincial, promulgado el 20 de marzo de 1925, confirió a las Corporaciones Provinciales la construcción y conservación de los mismos.

Paralelamente, se asiste a una mejora de la calidad de los principales itinerarios de carreteras del país. Mediante el Real Decreto-Ley de 9 de febrero de 1926 se da vía libre al Circuito Nacional de Firmes Especiales. Dentro del mismo se incluía el trayecto Ocaña-Albacete-Murcia-Cartagena, proyectándose a la vez el mejorar el ramal que desde la ciudad manchega conducía a Alicante. Por lo que

6 Vid. MORENO, F. «Caminos vecinales o vías secundarias», Revista de Obras Públicas, año LI, serie 7.a, t. 11, 1903, pp. 7 y ss.

7 ARCHIVO DIPUTACIÓN PROVINCIAL DE ALICANTE (ADPA), Memoria de la Diputación. Manuscrita, fechada el 30 de septiembre de 1903. 


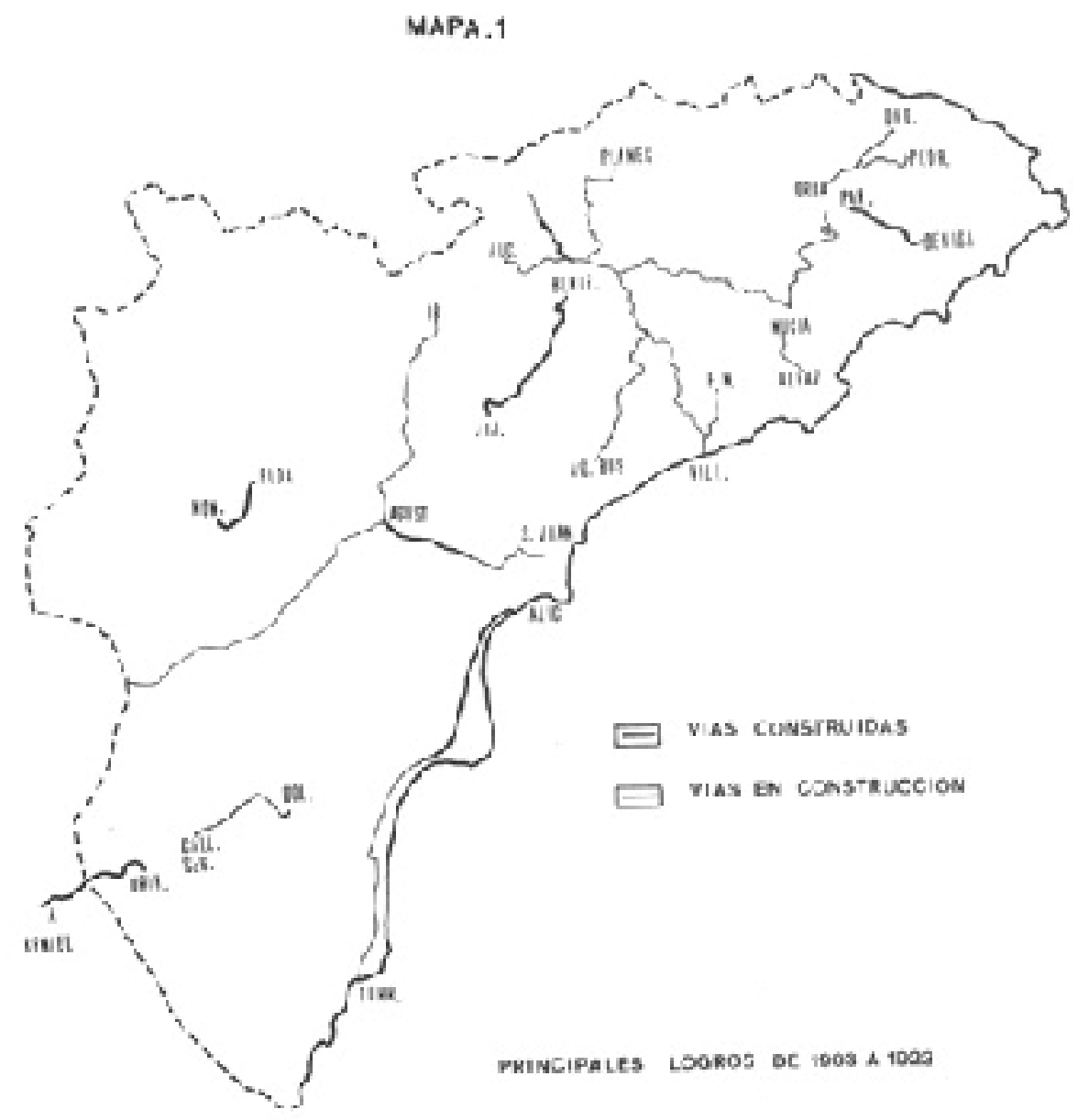

respecta a los circuitos transversales se contemplaba el eje Murcia-Alicante-Valencia.

También es destacable en el período, a nivel nacional, la Ley de 11 de julio de 1911 que suprimía el Plan de Carreteras de 1877, acusándole de inoperante, ya que el número de proyectos incluidos en el mismo sup eraban con creces las posibilidades de la Hacienda Pública, debido al electoralismo con que se planeaban las vías. Un nuevo Plan, el cuarto de los habidos en el país, era puesto en marcha por el Real Decreto de 5 de agosto de 1914.

\section{La red viaria alicantina en el período 1903-1923}

A nivel de la provincia alicantina, como fiel reflejo de lo que ocurría en la 
capital del Reino, las líneas directrices de la política de carreteras se centraban en la finalización de aquellos tramos, que siendo piezas claves de la red, estaban inconclusos, a la vez que se ponía esp ecial hincapié en el adensamiento progresivo de la malla secundaria, creando nuevos enlaces entre la misma y en la consecución de unos caminos vecinales transitables ${ }^{8}$.

En los primeros años del Reinado, el interés por dotar a la hoy a alcoyana de una buena accesibilidad se concreta en la continuación de la construcción de la vía que habría de unir la ciudad del Serpis con Callosa d'En Sarriá y de las del Barranco de la Batalla a Villajoy osa y de Planes a Relleu. El 23 de febrero de 1907, se abre a la circulación el viaducto de Alcoy y el 9 de diciembre se inaugura la carretera de Cocentaina a Benilloba9 .

En la Marina continúa la magna obra de la vía que unirá a Pego con Benidorm por Callosa, trabajándose en el tramo entre Bolulla y Tárbena, estando a punto de iniciarse el que discurre desde este último núcleo hasta Parcent. En 1918, se realiza el necesario puente sobre el río Jalón ${ }^{10}$. Las carreteras transversales de Villajoy osa a Finestrat, que se abrirá parcialmente al tráfico el 9 de diciembre de $1907^{11}$ y la que unirá la pista de Silla con Polop, por Alfaz del Pí y la Nucía, empiezan a hacerse realidad, mientras que se proy ecta enlazar la carretera de Alicante con Callosa por Altea la Vieja. Por otro lado, el 11 de diciembre de 1907 se inaugura parcialmente el camino de Ondara a Orba, mientras que se termina la que une el puente de Parcent con Benissa y se acomete la conexión de Benidoleig con Petrer.

En el Campo de Alicante, se trabaja en la variante de la carretera de Silla por Campello, y en distintos tramos de la que unirá la capital con Torrevieja, con trazado paralelo a la costa, abriéndose al tráfico el segundo trozo de la misma el 8 de noviembre de 1907. Por otro lado se brega en la que discurre hacia la Hoya de Castalla, habiéndose terminado la que conecta San Vicente con Agost. La accesibilidad de la Huerta alicantina también mejora al darle continuidad al camino que enlaza el Raspeig con Villafranqueza, Tángel y San Juan, a la vez que se tiende un nuevo enlace con la Marina desde Aguas de Busot a Relleu, que se abre parcialmente el 15 de julio de 1907, y se facilita el tránsito hacia la zona alcoyana p or la ruta entre Jijona y Benifallim $^{12}$.

${ }^{8}$ A principios de siglo, las carreteras dependientes del Estado en la provincia tenían una extensión de 638’5 kms., de los que 77'7 eran de primer orden; 222' 9 de segundo y 337' 9 de tercero; con una densidad de 40 kms. por cada 100.000 habitantes. Las dependientes de la Diputación sumaban 83’ 4, clasificadas como provinciales, a los que hay que sumar los referidos a caminos vecinales, que contabilizaban 124'5 kms., según MINISTERIO DE FOMENTO. Dirección General de Obras Públicas. Estadística de las Obras Públicas de España, carreteras y caminos vecinales. Madrid, Establecimientos Tipográficos Hijos de J. A. García, 1908.

-Para una detallada descripción de las carreteras y caminos existentes en la provincia a principios de siglo, vid. FIGUERAS PACHECO, F. «Provincia de Alicante», apud CARRERAS CANDI, F. Geografía General del Reino de Valencia. Barcelona, Alberto Martín, S. A., t. V, pp. 315-330. En el capítulo IV, fechado en 1913.

${ }^{9}$ ADP A, Vías y Obras (Vy O), Leg. 186.

${ }^{10}$ ADP A, V. y O., Leg. 474.

${ }^{11}$ ADP A, V. y O., Leg. cit. not. 9.

${ }^{12}$ ADP A, V. y O., Leg. cit. not. 9. 
En el Vinalopó, se pone en servicio el tramo que discurre por la provincia de la imp ortante carretera que enlaza Casas del Campillo con Villena por Onteniente, y se proyectan conexiones entre Villena y Bañeres, de Alcoy a Yecla y de la ciudad murciana al Pinoso, a través del puerto, abriéndose parcialmente en $1905^{13}$. Al mismo tiemp o se completan las conexiones de la comarca con Onteniente y Fuente la Higuera, inauguradas en parte en diciembre de 1907.

Un año antes, el ayuntamiento eldense elabora un plan de obras para prolongar hacia dicha ciudad la carretera entre Novelda y Monóvar ${ }^{14}$. Pero en 1913, las lluvias torrenciales caídas destrozan el puente de madera instalado para salvar el Vinalopó ${ }^{15}$, restableciéndose las comunicaciones en 1915, finalizando totalmente las obras de la vía en $1922^{16}$.

Una carretera que dará posibilidad de salida comercial a la agricultura de uno de los valles afluentes al corredor central del Vinalopó, es la que partiendo de Agost, y tras atravesar Monforte y Aspe, se adentra hacia los Hondones, discurriendo después por las partidas de Abanilla, de Macisvendas y Barinas, ya en la región murciana, para morir en la pista que, desde la estación de Archena, se dirige al Pinoso.

En el Bajo Vinalopó, se construye el camino que une Elche con la Vereda de Sendres, por el antiguo de la Huerta ${ }^{17}$, mientras que en la Vega Baja faltan por ejecutar doce kilómetros del que une Callosa de Segura a Dolores, que vertebra las tierras de la margen izquierda. Al otro lado del río, se trabaja en el que unirá Orihuela con su alfoz, para una vez cruzado el cauce, terminar en Almoradí. En estas fechas se hace también realidad el enlace de la capital comarcal con la localidad murciana de Beniel.

\section{La Dictadura de Primo de Rivera y los caminos vecinales}

La implantación de la Dictadura de Primo de Rivera va a suponer un importante relanzamiento de los caminos vecinales. El Reglamento de Vías y Obras, de 15 de julio de $1925^{18}$, que recogía lo promulgado por el Estatuto Provincial, de 20 de marzo, fijaba las bases de actuación en la materia. El 11 de febrero del siguiente año, la Corporación alicantina aprueba las bases para elaborar un Plan Provincial de Caminos Vecinales ${ }^{19}$.

${ }^{13}$ ADP A, V. y O., Leg. cit. not. 9.

${ }^{14}$ ARCHIVO MUNICIP AL DE ELDA, Acta del 8 de abril de 1906.

${ }^{15}$ ARCHIVO MUNICIP AL DE ELDA, Acta del 8 de septiembre de 1913.

${ }^{16}$ ARCHIVO MUNICIPAL DE ELDA, Acta del 3 de diciembre de 1922.

${ }^{17}$ ADP A, V. y O., Leg. cit. not. 9.

${ }^{18}$ ADP A, V. y O., Leg. 385.

19 ADP A, Acta del 11 de febrero de 1926.

-DIPUTACIÓN PROVINCIAL DE ALICANTE. Sección de Vías y Obras. Plan provincial de caminos vecinales. Concurso de subvenciones y anticipos. Alicante, Imprenta de J. Esplá, 1926.

-Es aprobado definitivamente por el Pleno de la Diputación el 5 de mayo de 1927. El mis mo recogía y seleccionaba los tramos más necesarios del extenso catálogo elaborado en 1926, Figuraban entre otras, obras tan necesarias como la de la vía que uniría La Aparecida, en la Vega Baja, con El Real, Llano de Brujas y Puente Tocinos en Murcia; o el que enlazaría Lorcha con Villalonga en la montaña interior. Esta última es aprobada para 


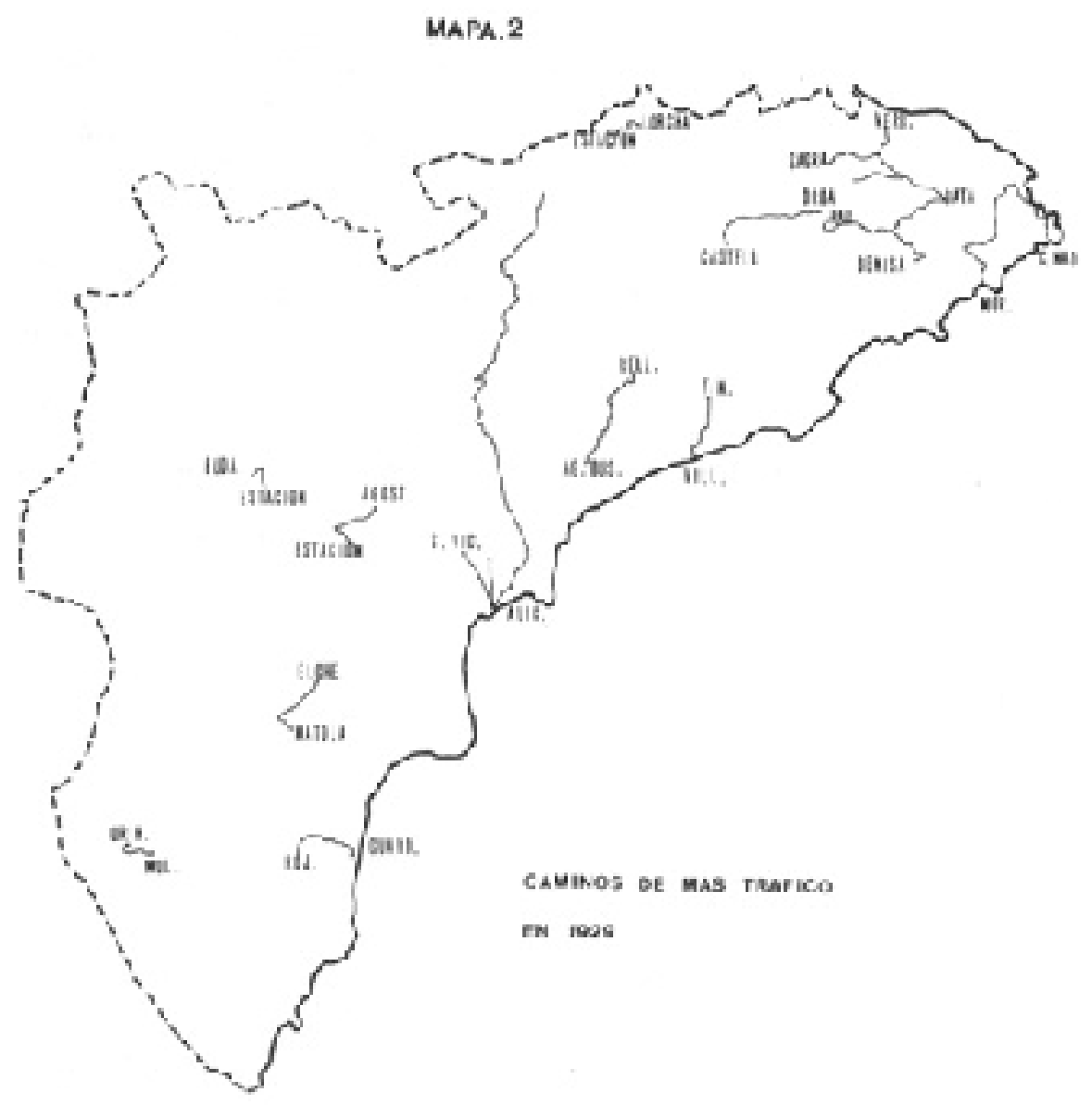

Durante 1927 se construyeron diez kilómetros y medio de caminos vecinales $^{20}$, destacando por su importancia el de Busot a las proximidades de Jijona ${ }^{21}$ y el de Torremendo a la estación del ferrocarril de Canteras en colaboración con la Diputación murciana ${ }^{22}$, a la vez que se acometen importantes reparaciones en

su realización en ese mismo año, según Acta del 15 de junio de 1926.

${ }^{20}$ ADPA, V. y O., Memoria relativa a los servicios efectuados por la Sección de Vías y Obras Provinciales en el año 1927.

${ }^{21}$ ADP A, V. y O., Leg. 484.

${ }^{22}$ ADP A, V. y O., Leg. 467. 
otros $^{23}$, consiguiéndose una red de 154 kilómetros de vías de este tipo, a los que se añadirían veinticuatro más, de nueva construcción, en $1928^{24}$.

A pesar del enorme esfuerzo realizado, los problemas de financiación estaban impidiendo cumplir el Plan de forma total, y a que el porcentaje de pago correspondiente a los ay untamientos se dilataba ampliamente, obligando a adoptar al Gobierno de la Nación un nuevo sistema de financiación mediante emp réstitos emitidos por las Corp oraciones Provinciales, separadamente o mediante Mancomunidad, acogiéndose el Consejo alicantino a la de Régimen Común, constituida el 25 de junio de 1928.

Ante las nuevas posibilidades crediticias, se elaboró un Plan Preferente y de Urgencia de Construcción de Caminos Vecinales ${ }^{25}$ que pretende terminar en un corto plazo de tiemp o los 72 kilómetros de vías pendientes de finalización. Los criterios de actuación eran de tres tipos: por un lado, sacar de su secular incomunicación a núcleos rurales de pequeña entidad ${ }^{26}$; por otro, mejorar la accesibilidad a distintas estaciones ferroviarias ${ }^{27}$, y, por un tercero, el acometer la prolongación de los caminos más transitados para dotarlos de may or funcionalidad ${ }^{28}$.

Sin embargo, a pesar de las nuevas vías de financiación, a partir de 1929, el ritmo de construcción de nuevos caminos decae notablemente ${ }^{29}$.

23 ADPA, V. y O., Leg. 291. Los principales caminos que se reparan son los de Alicante a San Vicente y Villafranqueza; de Aguas a Relleu; de Villajoyosa a Finestrat; de Orba a Ondara; de Rojales a Guardamar y de Molins a Orihuela.

${ }^{24}$ ADP A, V. y O., Leg. 385 y elaboración propia.

25 ADP A, V. y O., Plan preferente de urgencia de construcción de caminos vecinales, sufragados con los tres millones de pesetas que aproximadamente será lo que corresponda a la provincia de Alicante de los 300 millones que la Mancomunidad de Diputaciones consignará a este fin. Alicante, 12 de abril de 1928. Dicho plan fue aprobado el 14 de abril de 1928, pero la tramitación se prolongó hasta agosto (Acta del día 11), no siendo informado por la Jefatura de Obras Públicas hasta octubre. El placet definiti vo del Ministerio de Fomento no llegó hasta el 6 de junio del año siguiente (vid. leg. cit. not. 24).

${ }^{26}$ Los núcleos contemplados por el Plan son los de Miraflor, Alcocer de Planes, Benámer, Facheca, Asprillas, Balsares, Valverde Bajo, Rafal y la Matanza.

${ }^{27}$ Se intenta dar accesibilidad a las estaciones ferroviarias de Agres, San Juan (tren de vía estrecha) y Albatera-Catral.

28 Especialmente, los de Denia a Avesana y finalización del de Villena al Pinoso. Precisamente, a través del Informe del Ingeniero de Obras Públicas sobre el estado de los Caminos Vecinales, firmado en Alicante, a 17 de junio de 1929 (ADPA, V. y O.), conocemos las vías de este tipo con mayor tránsito. Los de mayor tráfico son los siguientes: de Alicante a San Vicente; de la capital a Villafranqueza; de Rojales a Guardamar; de Benichembla a Murla; de Vall de Laguart a Orba; de Benissa a Jalón, por Llíber; de Agost a su estación; de Teulada a Moraira y a Jávea; de Lorcha a su estación; de Pedreguer a Beniarbeig, Benidoleig y Gata; de Benissa a la estación; y de Jalón a Gata. Entre los muy transitados, pero con menor intensidad que los anteriores, se indican los siguientes: los que de Orihuela se dirigen a Arneva y a Molíns; Elche a Matola; Aguas a Relleu; Villajoyosa a Finestrat; Elda a su estación; Castells de Castells a Benichembla; puente de Parcent a Benissa; Sag ra a puente de Vergel; Moraira al embarcadero del Port; Jávea al cabo de la Nao; Benisili a la carretera de Cocentaina a Denia; y el que une la carretera de Játiva a Alicante con la de Cocentaina a Denia por Alquería de Aznar.

${ }^{29}$ ADP A, V. y O., Informe de Vías y Obras sobre la situación de los caminos vecinales de la provincia de Alicante. Alicante, 4 de noviembre de 1930. Este informe señala que 4 caminos están totalmente paralizados porque los ayuntamientos no abonan su parte; otros 4, siguen en construcción, pero a ritmo muy lento; y los proyectos de otras diez vías están aprobadas, pero no es posible iniciarlos por falta de presupuesto. 


\section{Precaria situación en la construcción de caminos en las postrimerías de la monarquía}

En los últimos meses del reinado de Alfonso XIII, la situación de ralentización en las obras de los caminos vecinales es un hecho, dado que los ay untamientos seguían sin hacer frente a sus compromisos. A mediados de 1930 se estaban ejecutando, con gran lentitud, varios de ellos, destacando el de Alcoray a a San Vicente, por la Cañada; el de Benillup a la carretera de Cocentaina a Denia; el de Jalón a Bernia, por Masarof; el de Benillup a la carretera de Planes a Relleu; y el de Puebla de Rocamora a la estación del ferrocarril de Almoradí-Dolores ${ }^{30}$. Al mismo tiempo, se está trabajando en el que unirá Vistahermosa con la Albufereta en la capital ${ }^{31}$ y se proyectan los de Muchamiel a la estación del ferrocarril de San Juan y el de la Murada a Barbarroja ${ }^{32}$.

Los problemas que conllevaba la construcción de caminos vecinales no se limitaban solamente a los atrasos en proveer fondos, sino también al choque de competencias con la Asociación General de Ganaderos, que protestaba airadamente por los perjuicios que se ocasionaban a la circulación pecuaria ${ }^{33}$.

\section{Dificultades de mantenimiento y conservación de la malla circulatoria}

Las dificultades morfológicas de la provincia y el régimen pluviométrico torrencial seguían siendo, como era usual en una larga y secular tradición, fuentes de quebrantos para la mayor parte de las vías, independientemente de su rango. Si en 1927, el ímp etu de las aguas arrastraba terraplenes y obras de fábrica, a la vez que se producían desprendimientos y descarnamiento del firme en los caminos rurales del área comprendida entre Benissa, Teulada, Benimantell y Jávea ${ }^{34}$, en 1930, la Diputación Provincial acuerda por unanimidad dirigirse a los poderes públicos estatales para que acometan el arreglo de vías tan importantes como la de Alicante a Alcoy y la de Ocaña, por estar en pésimas condiciones de conservación debido a causas climatológicas ${ }^{35}$.

Gran parte de la documentación estudiada sobre obras de conservación en el período, indica a la lluvia como principal agente de deterioro de la red viaria. Así, por ejemplo, en 1903, se ha de afrontar la reconstrucción parcial de la

${ }^{30} \mathrm{ADP}$ A, V. y O., Estado de las obras de construcción de caminos vecinales por la Excma. Diputación Provincial de Alicante. Alicante, 3 de septiembre de 1930.

${ }^{31}$ El proyecto en ADP A, V. y O., Leg. 286.

${ }^{32}$ ADP A., V.y O., Leg. cit. not. 23.

33 El presidente de la Asociación General de Ganaderos envió una carta al Ingeniero de Vías y Obras de la Diputación alicantina, fechada en Madrid, a 19 de noviembre de 1930, protestando por el hecho de que muchos caminos se estaban construyendo sobre vías pecuarias. estorbando, de esta forma, el paso del ganado. Apud ADPA, V. y O., Leg. cit. not. 23.

34 A D P A, V. y O., Informe del ingeniero de Vías y Obras al Presidente de la Diputación, fechado en Alicante, a 5 de julio de 1927.

${ }^{35}$ ADP A, Acta del 13 de noviembre de 1930. 
carretera de Alcoy a Bañeres, seriamente afectada por los fuertes temporales de noviembre del año anterior ${ }^{36}$.

Tres elementos básicos coadyuvaron a esta situación: por un lado, las dificultades orográficas de algunas zonas de la provincia; p or otro, el régimen pluviométrico de la zona, con lluvias de alta intensidad horaria; un tercero, la falta de calidad de la construcción, heredada ya del Ochocientos, que hunde sus raíces no solamente en la falta de presupuestos adecuados, sino también en deficiencias técnicas, tales como la ausencia, en la mayoría de los casos, de análisis previos geológicos, edafológicos, climáticos y de vegetación.

La escorrentía de las aguas ataca de forma notable a la red, máxime cuando la práctica totalidad de la misma, salvo contadas excepciones, estaba construida, en los mejores casos, con firme de macadam y, generalmente, se limitaban a ser caminos de tierra acondicionados.

Por ello, los aterramientos, desprendimientos y embarramiento del firme durante largos períodos de tiemp o eran usuales en nuestra red de carreteras y caminos. La situación era grave en las zonas montañosas sep tentrionales más difíciles, donde el abarrancamiento progresivo de las pendientes, por falta de un tratamiento específico de plantaciones vegetales o de obras de ingeniería, hacía usual la caída de derrubios sobre el trazado de las vías.

Los altos costos de mantenimiento de obras civiles de defensa contra las crecidas y la erosión excedían la realidad presupuestaria de los organismos competentes, por lo que la malla circulatoria vivía en constante deterioro, que solamente de forma parcial y, en la may oría de los casos, tardíamente se reparaba mediante la dotación de créditos extraordinarios.

La conservación de la red era sumamente difícil ya que los trazados no eran totalmente correctos. Es inusual encontrar canales de desagüe, zanjas, excavaciones absorbentes, sistemas de bombeo, correcciones de pendientes que tengan resuelto de forma eficaz el drenaje, y puentes y pontones con luz suficiente para dar salida a una circulación hídrica excepcional en cauces normalmente secos. De hecho, al observar trazados originarios de la ép oca, en algunos caminos se observan dos fenómenos: la constante interrupción de los drenajes tradicionales, mediante taponamiento de los mismos, y la creación de problemas de desagüe por la desnivelación que introduce el perfil transversal de la vía, con su clásico efecto barrera.

La realización de las obras públicas bajo la premisa del bajo costo en material para poder así aumentar la cantidad de mano de obra contratada con el fin de paliar el paro crónico, debido parcialmente a las profundas transformaciones que el capitalismo estaba introduciendo en el agro alicantino, es un elemento clave a la hora de enjuiciar las realizaciones del primer tercio del siglo XX.

Los bajos costos con que se proyectaron la mayor parte de los viales y la atrasada técnica aplicada en una buena parte de las obras de ingeniería que se acometieron, tuvieron una repercusión negativa en el funcionamiento de la red y en su mantenimiento eficiente. Una obra con escaso presupuesto supone modificar al mínimo la naturaleza, pero la acción antropógena realizada es suficiente

${ }^{36}$ ADP A, V. y O., Leg. 13. 
ciente para perturbar la normal escorrentía. Es frecuente en la zona septentrional del territorio provincial el que se hayan aprovechado para los trazados de las rutas terrestres las cuencas vertientes de pequeños valles y los barrancos de encajamiento vertical. Se utilizan de esta forma unas redes de fractura cuy as vertientes episódicamente poseen circulación hídrica, pero que en momentos de fuertes aguaceros, tienen un fuerte poder erosivo y de arrastre de derrubios que producen el escombramiento de los pavimentos y la ruptura de los tramos y obras de fábrica, impidiendo la circulación durante largos períodos de tiempo.

En otras áreas, el trazado de las carreteras busca, por más económico, las zonas más favorables de las cuencas vertientes, basadas en los puntos de menor desnivel en relación al perfil transversal y longitudinal del valle o las llanuras de inundación y conos de deyección, creando un típico fenómeno barrera, máxime cuando la luz de los arcos de desagüe es insuficiente a todas luces, taponando y cambiando de rumbo el devenir de las aguas en unos cauces, con frecuencia arreicos, pero conductores de grandes avenidas cuando se producen lluvias de máxima intensidad horaria.

\section{A modo de conclusión}

La p olítica de carreteras en el período alfonsino recoge la herencia del Ochocient os, centuria en la que se realizan los ejes viarios básicos de la provincia, a excepción de la ruta litoral meridional, todavía en construcción, y convierte al camino vecinal en protagonista principal de la red, que, mediante un sistema capilar cada vez más complejo, conecta el ferrocarril con ámbitos geográficos cada vez más lejanos a su trazado, al tiempo que, aprovechando la efervescencia tecnológica generada por el motor de explosión, intenta mejorar cualitativamente los viales básicos que unen la provincia con el exterior, como condición básica para que el nuevo sistema de transporte pueda fraguar y avanzar.

Y todo ello aprovechando el marco general de crecimiento económico, que no se verá realmente interrumpido hasta la llegada de los negativos efectos de la crisis del veintinueve, y de aumento de los efectivos demográficos de una provincia donde la vida urbana va tomando paulatinamente may or envergadura.

Un aumento de la productividad agrícola, con el auge del regadío y la renovación en los sistemas de explotación, unido a un creciente imp acto de la industria en ciertas áreas geográficas, sirve para que el desarrollo del transporte sea imprescindible en esta provincia, donde la economía comercial es un hecho y donde los negocios inmobiliarios y de construcción se acentúan al calor de un proceso paulatino de concentración capitalista, unido al impulso que las cap as intelectuales del país dan a la necesidad de reformar y desarrollar la atrasada faz del país.

Por todo ello, al finalizar el período e instaurarse la II Rep ública, la provincia de Alicante contaba con la suficiente infraestruct ura del transporte para que existiera una expansión del mercado interior y un afianzamiento de la industrialización, estando instalada plenamente en los circuitos capitalistas, como culminación del largo proceso histórico alentado por los prohombres liberales desde la época isabelina. 